\title{
A rapid and easy method for the DNA extraction from Cryptococcus neoformans
}

\author{
Fatma Mseddi, Mohammed Ali Jarboui, Amira Sellami, Hayet Sellami and Ali Ayadi*
}

\begin{abstract}
DNA isolation from C. neoformans is difficult due to a thick and resistant capsule. We have optimized a new and rapid DNA isolation method for Cryptococcus using a short urea treatment followed by a rapid method using a chelex resin suspension. This procedure is simpler than previously reported methods.
\end{abstract}

\section{Introduction}

Nucleic acid detection methods such as PCR have become a common tool for Cryptococcus neoformans species complex identification and diagnosis. Although PCR amplification can be performed directly from cultures, prior isolation of DNA is often preferred [1,2].

As the DNA extraction process eliminates many unknown interfering substances in the biological material, it plays an important role in ensuring consistent test results. DNA isolation from C. neoformans is difficult due to a thick and resistant capsule that is not readily susceptible to lyses.

Therefore, efficiency in the DNA extraction method using phenol, chloroform and isoamylic alcohol requires time and toxic solution manipulation, due to the organic solvents that may be hazardous to the environment and to the technician, and also several washing and centrifugation steps increasing the risk of sample contamination [3]. Several methods have been proposed as an alternative to the use of phenol and chloroform, such as commercial kits for DNA extraction. The use of kits offers a low risk of manipulation and they are faster than conventional protocols, but the amount of DNA recovered from the commercial kits is highly variable [4]

The objective of the present study was to compare four DNA extraction protocols from culture of collected strains from 2005-2009. This article summarizes the results of a comparison of the techniques in regards to good amplification and purity of obtained DNA.

\footnotetext{
* Correspondence: ali.ayadi@rns.tn

Laboratoire de Biologie Moléculaire Parasitaire et Fongique, Faculté de
} Médecine de, 3029, Sfax, Tunisia

(c) 2011 Mseddi et al; licensee BioMed Central Ltd. This is an Open Access article distributed under the terms of the Creative Commons Attribution License (http://creativecommons.org/licenses/by/2.0), which permits unrestricted use, distribution, and reproduction in any medium, provided the original work is properly cited.

\section{Materials and methods}

\section{Strains}

A total of 150 Tunisian Cryptococcus isolates from clinical and environmental strains and the following standard strains representing each serotype of $C$. neoformans H99 (serotype A), JEC 21 (serotype D), the hybrid IHEM 13877 (serotype AD), C. gattii Wm 276 (serotype B) and IHEM 4159 (serotype C) were used.

\section{DNA extraction}

Total genomic DNA was extracted from culture $\left(10^{8}\right.$ cells/ $\mathrm{ml}$ ) of Cryptococcus strains by means of the following 4 procedures: Protocol A used extraction with lyticase, phenol-chloroform and isoamylic alcohol; Protocol B used extraction with chelex, Protocol C used extraction using reagent kit (MasterPure yeast DNA purification KIT (Epicentre, Madison, USA)) and the new protocol D using urea chelex.

Protocol A: DNA extraction using lyticase, phenolchloroform and isoamylic alcohol

The method for DNA extraction from culture by phenolchloroform and isoamylic alcohol (Sigma-Aldrich, SP, Brazil) consisted of the suspension of cells recovered by centrifugation from $15 \mathrm{ml}$ of a shaken $(150 \mathrm{rpm}) 18 \mathrm{~h}$ YEPD culture in $2 \mathrm{ml}$ of SE (1.2 M sorbitol, o.1 M EDTA PH = 7.5) and following the method described by Shin-ichi [5]

\section{Protocol B: chelex DNA extraction}

DNA was extracted using a rapid method based on thermal shock and the chelation of components other than nucleic acids by using a resin suspension, as previously described [6] 
Protocol C: Extraction by kit (MasterPure yeast DNA purification KIT (Epicentre, Madison, USA))

The DNA extraction with kit (MasterPure yeast DNA purification KIT (Epicentre, Madison, USA)) was performed according to manufacturer instructions.

\section{Protocol D: new protocol using urea chelex}

Procedure includes the following steps.

(i) Cells recovered by centrifugation from $15 \mathrm{ml}$ of a shaken $(150 \mathrm{rpm}) 18 \mathrm{~h}$ YEPD culture were washed once with cold water, incubated $3 \mathrm{~h}$ in $2 \mathrm{ml}$ of urea buffer (urea $8 \mathrm{M}$, $\mathrm{NaCl} 0.5 \mathrm{M}$, Tris $20 \mathrm{mM}$, EDTA 20 mM, SDS 2\%, pH 8)

(ii) The pellet resuspended in $300 \mu \mathrm{l}$ of distilled water in a microcentrifuge tube. A volume of $100 \mu \mathrm{l}$ of Chelex solution (10\% Chelex-100 [Bio-Rad, Hercules, Calif.] in an aqueous solution of $0.1 \%$ SDS, $1 \%$ Nonidet P-40, and $1 \%$ Tween 80 ) was added. The tubes were incubated at $95^{\circ} \mathrm{C}$ for $30 \mathrm{~min}$ and then on ice for $5 \mathrm{~min}$. DNA was removed from the supernatant after $5 \mathrm{~min}$ of centrifugation $(10,000 \mathrm{rpm})$ and stored at $-20^{\circ} \mathrm{C}$ until used.

\section{DNA quantification}

DNA sample concentrations were determined by spectrophotometry at the wave length of $260 \mathrm{~nm}$ for the DNA and $280 \mathrm{~nm}$ for proteins, and the purity observed using OD 260/OD 280, in NanoDrop equipment (Thermo Fisher Scientific, Wilmington, DE, USA). Concentration results are given in $n g / \mu \mathrm{L}$, and the DNA purity results are reported as the OD 260/OD 280.

\section{DNA quality determination}

The DNA quality was accessed by electrophoresis and suitability for downstream application in RAPD analysis (Random Amplified polymorphic DNA). The quality of the DNA yielded by each method was determined by electrophoresing a $5 \mu \mathrm{l}$ sample in a $0.8 \%$ TBE-agarose gel, stained with ethidium bromide. To further demonstrate the quality of the extracted DNA.

The RAPD analysis was carried out using primer six (5'-CCCGTCAGCA-3') in a volume of $50 \mu \mathrm{l}$. The following cycle conditions were used: initial denaturation at $95^{\circ}$ $\mathrm{C}$ for five minutes, followed by 45 cycles of denaturation at $95^{\circ} \mathrm{C}$ for one minute, annealing at $36^{\circ} \mathrm{C}$ for one minute and amplification at $72^{\circ} \mathrm{C}$ for two minutes, and a final extension at $72^{\circ} \mathrm{C}$ for 10 minutes. Amplification products were separated by electrophoresis, on $2 \%$ agarose gels in $1 \times$ TBE buffer at $150 \mathrm{~V}$ for 2.5 hours and stained with ethidium bromide and then visualized under UV light.

\section{Results}

\section{Comparative analysis of DNA sample quantification}

Comparing DNA sample quantification from culture, we observed that the extraction with Protocol D gave the highest DNA concentration $(16,03 \mu \mathrm{g} / \mu \mathrm{l})$, as compared with Protocols A, B and C (Table 1). Therefore, Protocol
Table 1 DNA concentration and purity (OD 260/OD 280) obtained by the four DNA extraction techniques

\begin{tabular}{ccc}
\hline Protocols & $\begin{array}{c}\text { Average DNA concentration DNA }(\boldsymbol{\mu g} / \\
\boldsymbol{\mu l})\end{array}$ & $\begin{array}{c}\text { OD 260/OD } \\
\mathbf{2 8 0}\end{array}$ \\
\hline A & 4.29 & 1.715 \\
B & 8.36 & 1.78 \\
C & 7.54 & 1.685 \\
D & 16.03 & 1.75 \\
\hline
\end{tabular}

A presented a lower yield of DNA $(4.29 \mu \mathrm{g} / \mu \mathrm{l})$ than Protocols B and C (Table 1).

\section{Comparative analysis of DNA quality on agarose gel} Our results showed a variable yields and quality in DNA across the different extraction methods. Only the new method produced high yields that were of good quality DNA and we have obtained a clear DNA band when $10 \mu \mathrm{l}$ of the $400 \mu \mathrm{l}$ DNA preparation was run in an agarose gel $(0.8 \%)$ and stained with ethidium bromide (Figure 1$)$.

\section{Comparative Analysis of RAPD Profiles}

The results from DNA extractions compared by RAPD PCR generate a profile comprising many bands (Figure 2). The broad range of bands comprising community profiles was visible and intra- and inter-subject variations were readily observed with the new extraction Protocol D. Using the three other protocols (A, B and C), we demonstrated that there were many bands in the upper part of the RAPD gel that were not sufficiently resolved to describe differences (Figure 2).

\section{Discussion}

After experimenting with several DNA purification regimens, we have optimized a new and rapid DNA isolation method for Cryptococcus using a short urea treatment, described by Bolano and al. [7] with slide modifications, followed by a rapid method based on

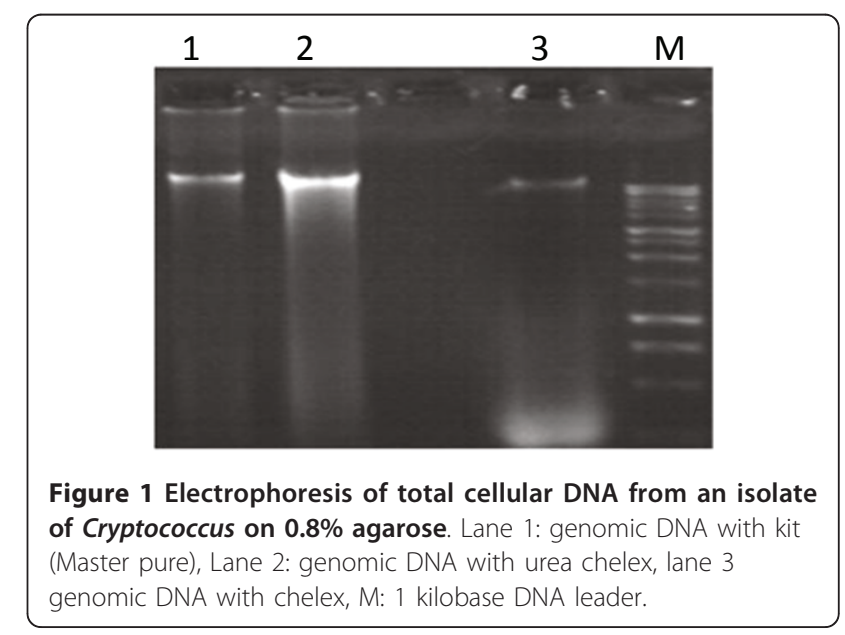




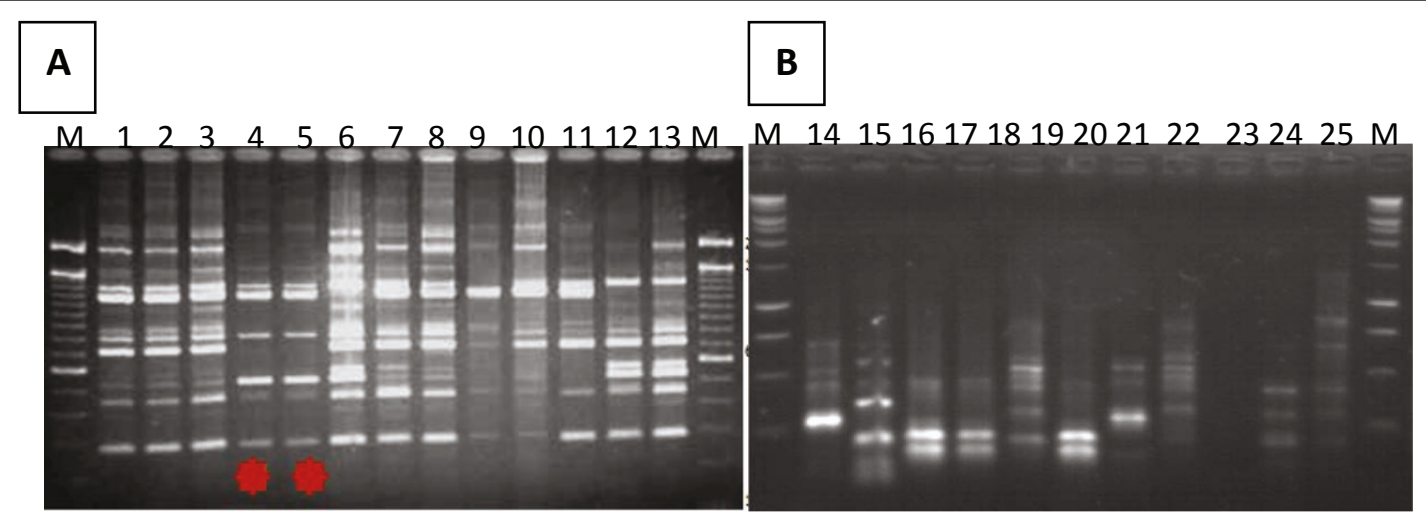

Figure 2 A comparison of RAPD profiles of C.neoformans. Example of environmental and clinical strains of C. neoformans amplified by PCR RAPD (lane 1-13 and lane 14-25) A- DNA was extracted using urea chelex B- DNA was extracted using chelex M: 100 pb DNA ladder.

thermal shock and the chelation of components, other than nucleic acids, using a resin suspension as previously described [6].

This new method is easier than described by Bolano et al. that incorporate a treatment with urea and bead beating [7]. The whole procedure can be completed within 4 hours. Up to now, we have used the technique to isolate DNA from 150 Tunisian Cryptococcus isolates from clinical and environmental strains. In addition, we also have succeeded in generating sufficient DNA from Cryptococcus isolates using this rapid method. We have obtained a clear DNA band when $10 \mu \mathrm{l}$ of the $400 \mu \mathrm{l}$ DNA preparation was run in an agarose gel $(0.8 \%)$ and stained with ethidium bromide.

Further, quantity and quality of DNA extract was influenced by the extraction method.

Data from this study indicate that the new DNA extraction method using urea chelex produced high quality DNA that can be amplified using PCR RAPD for comparisons of C. neoformans fingerprint profiles. These observations demonstrate that optimal DNA yield from culture is obtained by this method. Higher extraction efficiency allows for better recovery of DNA from an environmental sample resulting in a more comprehensive profile of $C$. neoformans. On the other hand, poor DNA extraction may lead to a poor PCR RAPD profiles. Higher DNA yield also increases recovery of DNA from C. neoformans isolate and chances of detecting polymorphism.

This procedure modifies and considerably simplifies previously reported methods for extraction of DNA.

It is likely that this procedure could be applied to the extraction of many other fungal cultures and, possibly, clinical specimens. It provides a rapid, reliable, and lowcost alternative to the existing DNA purification protocols used in research and clinical laboratories. The availability of this DNA extraction procedure for Cryptococcus not only would reduce the workload considerably but also would decrease the test turnaround time.

\section{Authors' contributions}

MF conceived the method, designed and carried out the validation of the study and writes the manuscript. MAJ participated in execution of the study. SA and AA designed the study and contributed to writing the manuscript. All authors contributed to writing the manuscript. All authors read and approved the final manuscript.

\section{Competing interests}

The authors declare that they have no competing interests.

Received: 25 February 2011 Accepted: 21 July 2011

Published: 21 July 2011

\section{References}

1. De Baere T, Claeys G, Swinne D, Verschraegen G, Muylaert A, Massonet C, Vaneechoutte M: Identification of cultured isolates of clinically important yeast species using fluorescent fragment length analysis of the amplified internally transcribed rRNA spacer 2 region (ITS2). BMC Microbiol 2002, 25:21.

2. LuO G, Mitchell TG: Rapid identification of pathogenic fungi directly from cultures by using multiplex PCR. J Clin Microbiol 2002, 40:2860-2865.

3. Fernandes JV, Meissner RV, Fernandes TAAM, Rocha LRM, et al: Comparação de três protocolos de extração de DNA a partir de tecido fixado em formol e incluído em parafina. J Bras Patol Med Lab 2004, 40: 141-146.

4. Löffler J, Hebart H, Schumacher U, Reitze $H$, et al: Comparison of different methods for extraction of DNA of fungal pathogens from cultures and blood. J Clin Microbiol 1997, 35:3311-3312.

5. Shin-ichi F, Takuma H: DNA fingerprinting patterns of Candida species using Hinf1 endonuclease. Intern J Syst Evol Microbiol 2000, 50:1381-1389.

6. Hennequin $C$, et al: Identification of Fusarium species involved in human infections by 28S rRNA gene sequencing. J Clin Microbiol 1999, 37(11):3586-3589.

7. Bolano A, Stinchi S, Preziosi R, Bistoni F, Allegrucci M, Baldelli F, Martini A, Cardinali G: Rapid methods to extract DNA and RNA from Cryptococcus neoformans. FEMS Yeast Res 2001, 1:221-224.

doi:10.1186/1480-9222-13-5

Cite this article as: Mseddi et al:: A rapid and easy method for the DNA extraction from Cryptococcus neoformans. Biological Procedures Online 2011 13:5 